\title{
Reticulação Progressiva de Pericárdio Bovino com Glutaraldeído para Confecção de Válvulas Cardíacas Biológicas.
}

\author{
Gilberto Goissis, Sônia D. Figueiró, Domingo M. Braile, Renato B. de Araujo e Vladimir D. A. Ramirez
}

Resumo: Este trabalho descreve um novo processo para a reticulação de matrizes de colágeno com glutaraldeído para a preparação de materiais para a confecção de válvulas cardíacas biológicas, e consiste no tratamento do pericárdio bovino com concentrações progressivamente crescentes com este reagente no intervalo de concentração entre 0,005 e 0,5\% em tampão fosfato, $\mathrm{pH} 7,4$. O perfil da reação com glutaraldeído, quando comparado com o procedimento convencional, foi mais homogêneo e os materiais obtidos apresentaram propriedades térmicas e mecânicas similares. Entretanto, mostraram-se significativamente mais estáveis à degradação enzimática, principalmente em relação à tripsina $(17 \mathrm{x})$, que é devido provavelmente à formação de ligações de reticulação mais homogêneas e/ou em maior densidade. Os resultados mostram que metodologia seqüencial estudada, associada ao tratamento convencional, pode constituir-se em uma técnica de pré-tratamento promissora para a preparação de materiais mais eficientes para confecção de biopróteses.

Palavras-chave: Pericárdio bovino, reticulação progressiva, glutaraldeído, biopróteses

\section{Introdução}

Os tratamentos de tecidos biológicos ricos em colágeno, tais como o pericárdio bovino (PB) e válvulas porcinas, com agentes de reticulação que incluem o glutaraldeído (GA), utilizados durante o processo de fabricação de válvulas cardíacas (biopróteses) tem como objetivos principais, a redução das propriedades antigênicas, por reagir com resíduos celulares, proteínas e outros materiais sensibilizantes e, melhorar suas propriedades mecânicas, pela introdução de ligações de reticulação ${ }^{[1]}$. Apesar deste tratamento, estes materiais, que são utilizados para a confecção dos folhetos das válvulas, entre outros problemas estatisticamente menos significativos, após 7 anos pós-implante ${ }^{[2]}$ sofrem um processo de calcificação degenerativa, que têm origem nas micro trincas ${ }^{[3]}$ geradas pela solicitação mecânica de suas superfícies, prejudicando sensivelmente o mecanismo de abertura e fechamento das biopróteses. A consequiência, é a redução progressiva da performance hidrodinâmica até sua falha comple$\operatorname{ta}^{[2-4]}$. Procedimentos alternativos à reticulação com glutaraldeído, como forma de solucionar este problema, incluem o pré-tratamento com agentes tensoativos, a extração do PB com solventes orgânicos e o uso de anticalcificantes ${ }^{[5,6]}$, ou ainda novos métodos de reticulação com compostos funcionalizados com grupos epóxi ${ }^{[7]}$ azidas $^{[8]}$ diisocianatos ${ }^{[9]}$ e carbo-

Gilberto Goissis - Depto. Quím. Fís. Molec., IQSC-USP, Av. Dr. Carlos Botelho 1465, Cx. P. 780, São Carlos, CEP 13560-970, SP; Sônia D. Figueiró Departamento de Química Analítica e Físico-Química, Universidade Federal do Ceará; Domingo M. Braile, Renato B. de Araujo, Vladimir D. A. Ramirez - DPP - Braile Biomédica S.A., São José do Rio Preto, SP. 
diimidas ${ }^{[10]}$, ou ainda, o tratamento com ácido $\alpha$-aminoolêico, principalmente de válvulas porcinas ${ }^{[11]}$. Procedimentos alternativos utilizando-se do próprio glutaraldeído também têm sido propostos e incluem a fixação dinâmica ${ }^{[12]}$, o uso de temperaturas mais elevadas $^{[13]}$ ou ainda o tratamento com o glutaraldeído protegido na forma de acetais ${ }^{[14]}$.

Quimicamente, a ação do glutaraldeído sobre matrizes colagênicas está relacionada com sua capacidade de reagir com $\varepsilon$-amino grupos de resíduos dos amino ácidos lisina e hidroxilisina da proteína para formar ligações cruzadas do tipo base de Schiff $(-\mathrm{CH}=\mathrm{N}-)^{[15]}$. Entretanto estas reações não são homogêneas devido à heterogeneidade do tecido ${ }^{[16]}$ e à química complexa do GA em soluções aquosas (Figura 1) ${ }^{[17]}$. Algumas das resultantes são a impermeabilização superficial do tecido (Figura 2) ${ }^{[18]}$ e a formação de ligações de reticulação poliméricas do tipo poli-GA (Figura 2) ${ }^{[19]}$. Para o caso do tecido hepático, foi mostrado que a penetração do GA é inversamente proporcional à espessura da $\operatorname{amostra}^{[20]}$ e para o $\mathrm{PB}$, a reticulação ocorre apenas nas fibras superficiais, principalmente quando em altas concentrações, condição utilizada no processamento do $\mathrm{PB}$ utilizado na confecção de biopróteses, que é de $0,5 \%$ [21].

Essa impermeabilização para o PB é um processo rápido e está associada à formação de ligações cruzadas poliméricas do tipo poli-GA (Figura 2). Como resultante, obtém-se regiões internas da matriz não reticuladas e menor biocompatibilidade em relação ao material superficial. Estas regiões parcial ou to-

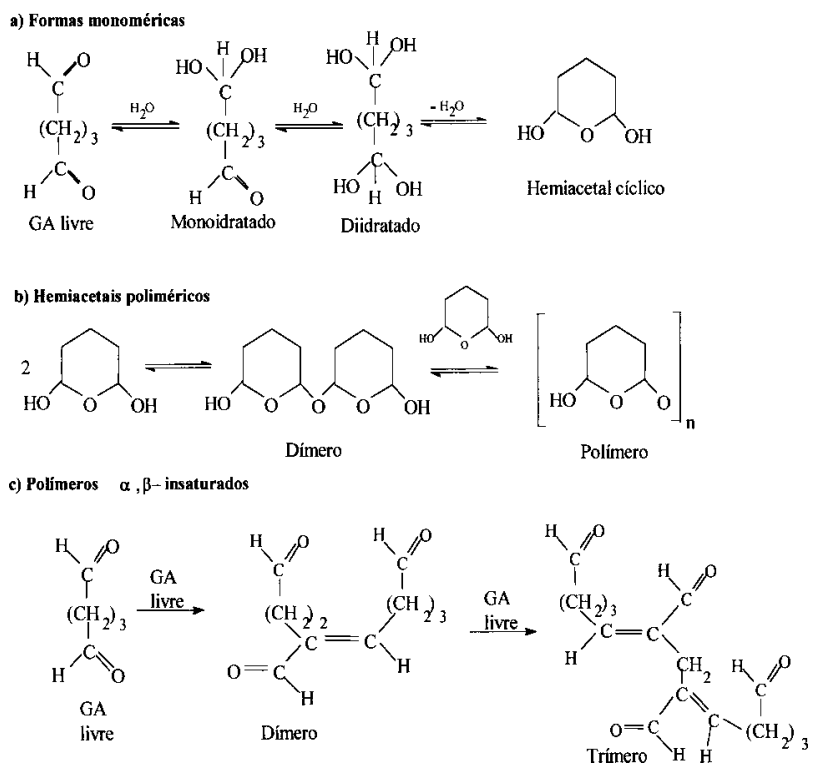

Figura 1. Esquema representativo da heterogeneidade química do glutaraldeído em solução aquosa.

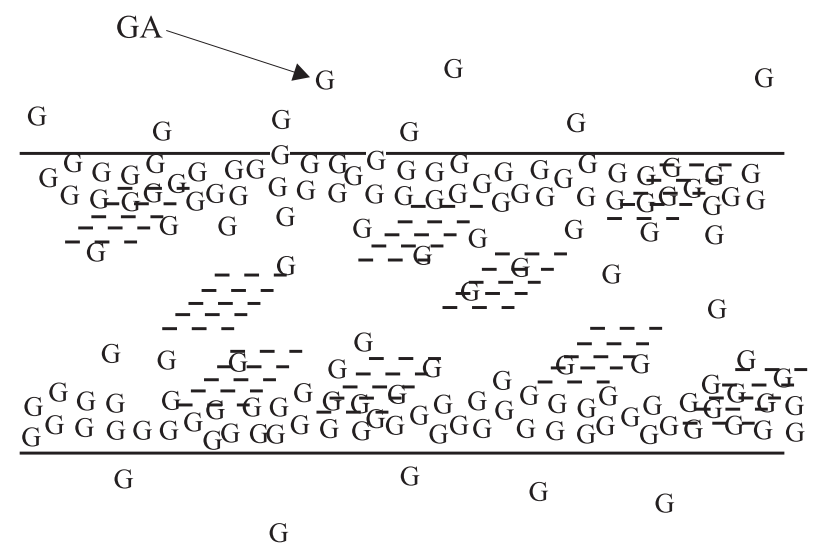

Figura 2. Diagrama ilustrativo da impermeabilização de matrizes de colágeno induzida pelo tratamento com soluções de glutaraldeído em concentrações elevadas.

talmente não reticuladas, após exposição devido à formação das trincas, funcionam como sítios primários de calcificação ${ }^{[3]}$. Além desta impermeabilização, as ligações cruzadas poliméricas ${ }^{[19]}$ tendem, pós-implante, a liberar no sistema vascular o GA na sua forma livre, resultando em manifestações de toxicidade sistêmica $^{[22]}$. Tratamentos com produtos capazes de reagir com a função aldeídica, tais como o ácido glutâmico $^{[23]}$, não minimizam estes problemas visto que apenas neutralizam as funções aldeídicas livres do GA, não impedindo a liberação a partir das ligações cruzadas poliméricas presentes.

Tendo em vista que incrementos de temperatura associado a baixas concentrações de GA promovem uma maior difusão do reagente na matriz ${ }^{[14]}$, o objetivo deste trabalho foi o desenvolvimento de uma metodologia de reticulação para a obtenção de materiais mais homogêneos, baseado no tratamento do $\mathrm{PB}$ com concentrações inicialmente menores e, progressivamente crescentes até aquelas convencionalmente utilizadas ${ }^{[21]}$. A expectativa é introduzir na matriz ligações de reticulação mais homogêneas e a redução intensidade do processo de impermeabilização por diminuição das ligações cruzadas poliméricas e, como conseqüência, a redução da calcificação degenerativa, um dos maiores problemas observados com as biopróteses pós-implante.

\section{Parte Experimental}

O PB utilizado, fornecido pela Braile Biomédica S.A., foi coletado de animais abatidos com idades entre 30 e 60 meses e, antes de qualquer tratamento químico foi lavado previamente com solução de $\mathrm{NaCl}$ 
0,9\%, água e equilibrado em soluções aquosas de etanol com concentrações crescentes entre 10 a 70\%. Para realização dos ensaios de reticulação a concentração de etanol foi reduzida gradualmente de modo similar, seguido de equilíbrio em solução tampão fosfato (TF) 0,13mol/L pH 7,4. O GA utilizado (Union Carbide) foi previamente purificado com carvão ativado ${ }^{[21]}$. A relação para as absorbâncias $285 / 235 \mathrm{~nm}$ foi de 6,42 , comparado com um valor mínimo aceitável de $1,50^{[21]}$.

\section{Reticulação com GA}

Não Seqüencial: Peças de PB, foram tratadas em TF, com concentrações de GA de 0,001\%, 0,005\%, $0,05 \%$ e $0,5 \%$ por tempos que variaram de 0 a 180 min. seguido pelo tratamento com uma solução de glicina:borato, 0,025M:0,05M, pH 9,2, durante 10 minutos e TF, onde foram mantidos para os ensaios subsequentes.

Seqüencial: PB limpo e previamente equilibrado em TF $0,13 \mathrm{M} \mathrm{pH} \mathrm{7,4,} \mathrm{foi} \mathrm{cortado} \mathrm{em} \mathrm{peças} \mathrm{de} 5 \mathrm{~cm}^{2}$, que foram tratadas cada uma, à temperatura ambiente, com $30 \mathrm{~mL}$ de soluções diluídas de GA em concentrações que variaram de $0,001 \%$ a $0,5 \%$ por intervalos de tempo de 0 a $180 \mathrm{~min}$. Ao final dos tempos de tratamento pré-estabelecidos, as peças foram tratadas com uma solução de glicina:borato, 0,025M:0,05M, pH 9,2, durante 10 minutos. A seguir foram lavadas com TF onde foram mantidos para os ensaios subsequentes. Os experimentos de reticulação com GA foram feitos como descritos a seguir:

Experimento A - Quatro conjuntos de peças de PB foram tratadas separadamente com GA nas concentrações $0,001 \%, 0,005 \%, 0,05 \%$ e $0,5 \%$, sendo que cada peça foi removida nos tempos de 0 a 180 minutos para determinação da temperatura de encolhimento (Ts).

Experimento B - Peças de PB foram tratadas com solução de GA $0,05 \%$ durante os tempos de 0 a 180 minutos para os ensaios de inchamento.

Experimento $\mathrm{C}$ - Peças de $\mathrm{PB}$ foram tratadas com solução de GA a $0,001 \%$ durante 3 horas e a seguir distribuídas igualmente em três conjuntos que foram tratados com GA nas concentrações $0,005 \%, 0,05 \%$ e $0,5 \%$. As peças foram removidas de tempos em tempos, dentro do intervalo 0 e 180 minutos para determinação de Ts.

Experimento D - Peças de PB foram tratadas seqüencialmente por tempos de 24 horas com soluções de GA $0,001 \%$ e $0,005 \%$ seguido de tratamento com GA $0,05 \%$ e $0,5 \%$ separadamente. Cada peça foi removida nos tempo de 0 a 180 minutos para os ensaios de Ts.

Experimento E - Peças de PB foram divididas em dois conjuntos, um dos quais recebeu o tratamento com GA $0,05 \%$, e o outro o tratamento seqüencial por tempos de 24 horas com GA nas concentrações $0,001 \%$ e $0,005 \%$, seguido de tratamento com GA $0,05 \%$ por 3 horas. Cada peça foi removida de cada conjunto nos tempos de 0 a 180 minutos para os ensaios de hidrólise com tripsina.

\section{Estabilidade Térmica}

Temperatura de encolhimento (Ts): Foi determinada em um equipamento de ponto de fusão (Quimis, modelo Q 340-13), adaptado para Ts, utilizando-se amostras de PB de 2,0 x 0,2 cm, preparada como descrito acima, e que foram previamente equilibradas em TF. A taxa de aquecimento foi de $2,0^{\circ} \mathrm{C} / \mathrm{min}$ no intervalo de 20 a $100^{\circ} \mathrm{C}$. Os valores de Ts (média de 4 determinações), foram obtidos com as amostras imersas em tampão fosfato $0,14 \mathrm{~mol} / \mathrm{L}, \mathrm{pH} 7,4$, colocadas em um tubo de pirex graduado de $20 \mathrm{~mm}$ imerso em banho de óleo de silicone.

Temperatura de desnaturação (Td): Foi determinada em um aparelho Du Pont DSC 910 acoplado a um terminal Du Pont modelo 9900, calibrado com padrão de índio. Peças de pericárdio de $0,5 \mathrm{~cm}$ de diâmetro, pesando entre 8,7 a 10,1 mg (peso seco), foram seladas em capsulas de alumínio e aquecidas a uma razão constante de $5^{\circ} \mathrm{C} / \mathrm{min}$ de 20 a $120^{\circ} \mathrm{C} \mathrm{em}$ atmosfera de $\mathrm{N}_{2}$.

\section{Ensaios de Inchamento}

De cada peça de pericárdio bovino tratada com GA como descrito no experimento $\mathrm{B}$, foram removidos 3 discos de $0,5 \mathrm{~cm}$ de diâmetro que foram equilibrados em solução TF por 12 horas em uma temperatura de $5^{\circ} \mathrm{C}$. Após este período, cada conjunto de três discos foi introduzido em tubo de pirex previamente tarado, o material congelado em nitrogênio liquido seguido de liofilização até peso constante. Os resultados de inchamento foram expressos em massa de água por massa de tecido seco.

\section{Hidrólise Enzimática}

- Colagenase: De cada peça de PB tratada ou não com GA, como descrito no experimento $\mathrm{E}$, foram removidos quatro discos, cada um com $0,5 \mathrm{~cm}$ de diâ- 
metro. Cada disco foi introduzido em um tubo de pirex, o material congelado em nitrogênio líquido, liofilizado até peso constante (peso aproximado de cada disco foi em torno de $3 \mathrm{mg}$ ), e a seguir tratado com $3 \mathrm{ml} \mathrm{de}$ solução de colagenase em tampão Tris $10 \mathrm{mM} \mathrm{pH} \mathrm{7,4}$ à $37^{\circ} \mathrm{C}$. Para cada dois dos quatro discos de cada conjunto, foram usadas diferentes relações de massa de enzima:proteína, ou seja, de 36,25 e 72,5 U de enzima/ mg de colágeno respectivamente e, antes de serem colocados na estufa, os tubos foram submetidos a vácuo e mantidos em dessecador nesta condição por $1 \mathrm{~h}$ para garantir uma distribuição homogênea da enzima no material. Após 72h, o colágeno residual, na forma de discos, foi removido e a solução centrifugada à 3000 rpm por 10 minutos. As soluções sobrenadantes foram congeladas, liofilizadas e o resíduo hidrolizado para a determinação da quantidade de colágeno solubilizado por meio da hidroxiprolina ${ }^{[24]}$.

- Tripsina: Discos de PB tratados com GA e com um peso médio em torno de $3 \mathrm{mg}$, foram previamente desnaturados por aquecimento em banho-maria por 2 min. Cada disco foi tratado com tripsina (Merck$3,5 \mathrm{U} / \mathrm{mg}$ ) dissolvida em tampão borato $0,2 \mathrm{M} \mathrm{pH}, 7,6$ a $23^{\circ} \mathrm{C}$ durante 10 horas, usando uma relação de enzima:proteína de $0,056 \mathrm{U} / \mathrm{mg}$ de PB. Após a hidrólise, cada disco foi removido da solução, e esta aquecida em banho-maria seguido de centrifugação a 3000 rpm por 10 min. A análise do grau de solubilização dos peptídeos foi realizada pelo método Biureto ${ }^{[25]}$.

\section{Ensaios Mecânicos}

Os PB utilizados nos ensaios mecânicos, antes da fixação com GA, foram fixos em bastidores de plásticos de $16 \mathrm{~cm}$ de diâmetro com tensão controlada, a fim de evitar dobras ou estiramento excessivo do tecido. Os materiais estudados foram: a) - PB tratado, seqüencialmente, na concentração de GA de 0,001 e $0,05 \%$ e b) - PB tratado por processo convencional para a obtenção de materiais para a confecção de válvulas cardíacas. Todos estes tratamentos foram realizados pela Braile Biomédica S.A, em uma temperatura de $25^{\circ} \mathrm{C}$ e segundo a norma ASTM-D-638 da American Society for Testing and Materials ${ }^{[21]}$.

\section{Resultados e Discussão}

As curvas de estabilidade térmica determinada como Ts para os materiais resultantes do tratamento

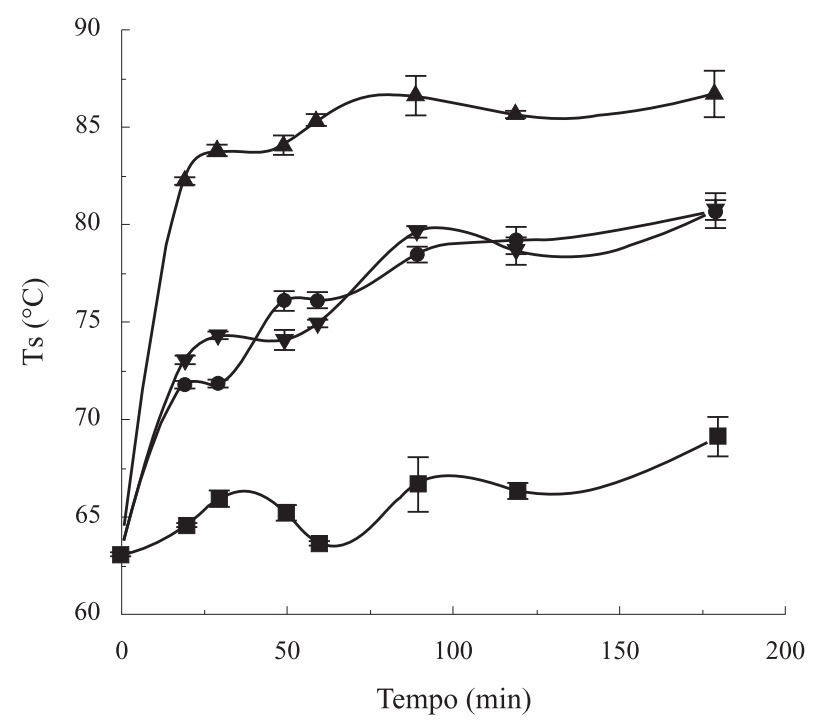

Figura 3. Comportamento da estabilidade térmica (temperatura de encolhimento) de pericárdio bovino tratado com glutaraldeído com

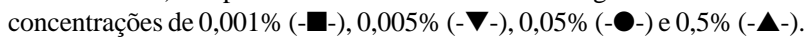

do pericárdio bovino com soluções de GA de concentrações de 0,001 a $0,5 \%$, processo não progressivo (Figura 3), mostraram um comportamento bastante irregular, caracterizado pela presença de pontos de máximos e mínimos para todas as concentrações utilizadas, cujas posições e intensidade foram dependentes da concentração de GA utilizada.

Para a menor concentração de GA utilizada, $0,001 \%$, os efeitos foram mais acentuados, com pontos de máximos observados após 30 e 90 min de reação e com valores de Ts de respectivamente $65,9 \pm 0,4$ e $66,5 \pm 1,4^{\circ} \mathrm{C}$. Um ponto de mínimo para Ts foi observado após 60 min de reação e seu valor foi de $63.2 \pm 0.1^{\circ} \mathrm{C}$. Como as ligações do tipo de bases de Schiff formadas entre $\varepsilon$-amino grupos de lisina ou hidroxilisina com GA, são estáveis nas condições para a determinação de $\mathrm{Ts}^{[17-19]}$, não é provável que as variações observadas na estabilidade térmica do PB sejam resultantes de uma diminuição do número de ligações cruzadas. Estas alterações, como sugerido pelo comportamento da curva de inchamento do PB tratado com GA a $0,5 \%$ (Figura 4), parecem ser resultantes de modificações estruturais da matriz colagênica, pois Ts mantêm uma correlação inversa com o grau de inchamento ${ }^{[26]}$. Embora para os tempos de reação de 20 e 30 min de reação a redução observada de $2,71 \mathrm{~g}$ água/g tecido para 2,08 g água/g tecido seja o comportamento esperado em função do aumento do grau de reticulação, à medida que a reação prosseguiu, um outro ponto de máxima foi observado após $90 \mathrm{~min}$, com um valor de 2,97 g água/g tecido. A este ponto de máxi- 


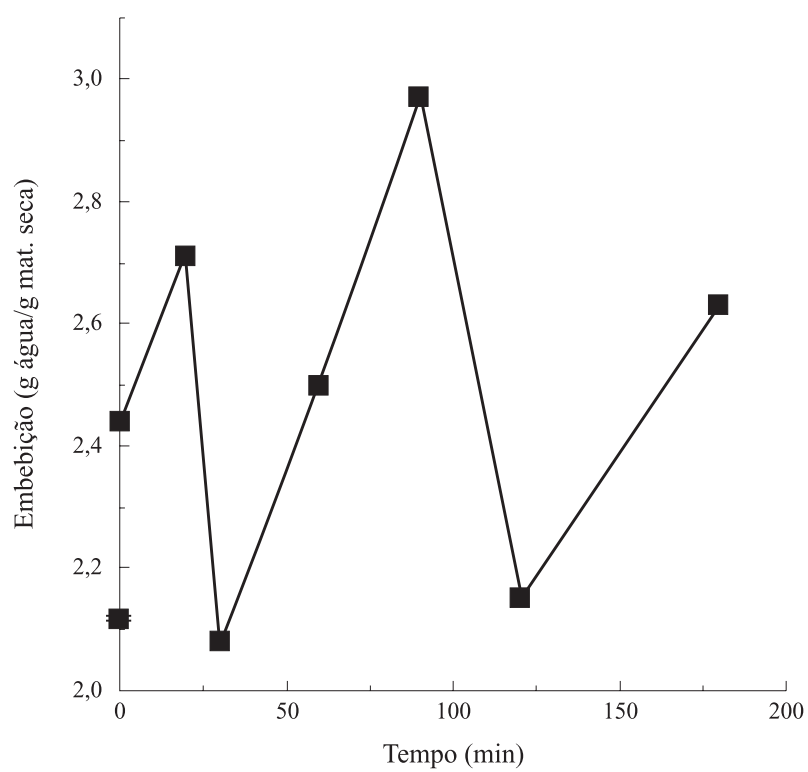

Figura 4. Comportamento para o inchamento do pericárdio bovino em função do tempo de reação para com uma solução tamponada de GA a $0,05 \%$

mo, um novo ponto de mínimo foi detectado e com um valor 2,31 g água/g tecido (Figura 4). Na reação do PB com GA, o grau de inchamento é função de duas variáveis que operam em sentidos opostos: uma na qual o grau de inchamento deveria decrescer em função diminuição dos espaços ocasionados pelo aumento da reticulação; a outra, um aumento desta variável, também função da progressão da reação de reticulação, como resultado do aumento da resultante da carga negativa da matriz a pH fisiológico, devido ao equilíbrio Donnan, pois um mol de GA consome duas unidades de carga positiva correspondentes a $\varepsilon$-amino grupos de lisina ou hidroxilisina ( $\mathrm{pH}$ do colágeno $=6,8-7,1$ ). Apesar da dificuldade de qualquer previsão de comportamento para o grau de inchamento (aumentos ou diminuições), a presença de pontos de mínimos e de máximos, tanto para Ts quanto para o grau de inchamento, dificilmente poderiam ser explicados por estes efeitos antagônicos, a menos que sejam devido alterações estruturais significativas da matriz do PB que estejam ocorrendo em função do progresso da reação, principalmente sabendo-se que no envelhecimento do tecido conjuntivo animal, que é uma conseqüência do aumento do grau de reticulação da matriz, Ts e grau de inchamento mantém entre si uma relação inversa ${ }^{[26]}$.

A heterogeneidade da reação do PB com GA foi confirmada também por DSC para materiais tratados com concentrações de 0,05 e $0,1 \%$.(Figura 5). Para o PB tratado com GA a $0,05 \%$ os perfis de DSC (Figura 5a) obtidos em função do tempo de reação, mos-
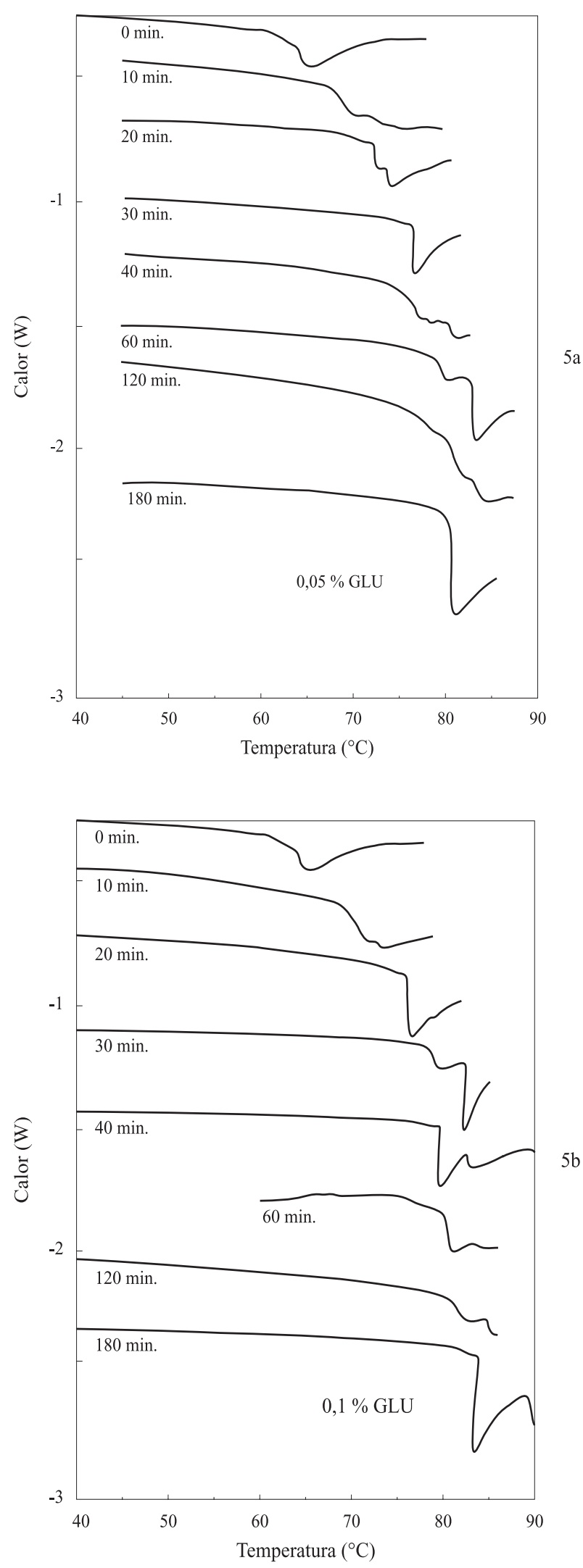

Figura 5. Comportamento da estabilidade térmica (DSC) de pericárdio bovino em função do tempo de reação e concentração de GA: a) - 0,05\%; b) $-0,1 \%$. 
traram uma regressão estrutural (diminuição do valor de Td) entre os tempos de reação 60 e 180 min. Neste caso o valor de Td para as transições mais importantes variaram de $83,0^{\circ} \mathrm{C}$ para o tempo de $60 \mathrm{~min}$, decrescendo para $80,6^{\circ} \mathrm{C}$ para o tempo de reação de 120 min (Figura 5a). Resultados similares foram observados nos perfis das curvas de DSC para o tratamento de PB com uma concentração de GA de $0,1 \%$ (Figura $5 b$ ), exceto que a regressão da transição foi observada entre tempos de 40 e $60 \mathrm{~min}$. Os valores de Td para as transições mais intensas foram de 82,3 e $78,9^{\circ} \mathrm{C}$ respectivamente. Estes resultados, assim como aqueles da Figura 3, mostram também que a heterogeneidade da reação depende também da concentração de GA.

Os resultados acima estão então de acordo com aqueles já descritos anteriormente na literatura, exceto que para a tensão de deformação ${ }^{[27]} \mathrm{e}$ intensidade da calcificação determinada para PB em implantes subcutâneos ${ }^{[28]}$, onde também foram observados pontos de máximo e de mínimo em função do tempo de reação e concentração de GA utilizados na reação de reticulação.

Os resultados para o comportamento da estabilidade térmica de PB tratados pelo método seqüencial, isto é a fixação com concentrações progressivamente maiores de GA de $0,001 \%, 0,005 \%, 0,05 \%$ e $0,5 \%$ são mostrados pela Figura 6. Como característica principal, em relação ao tratamento não seqüencial (Figura 3), pode ser observada uma redução acentuada

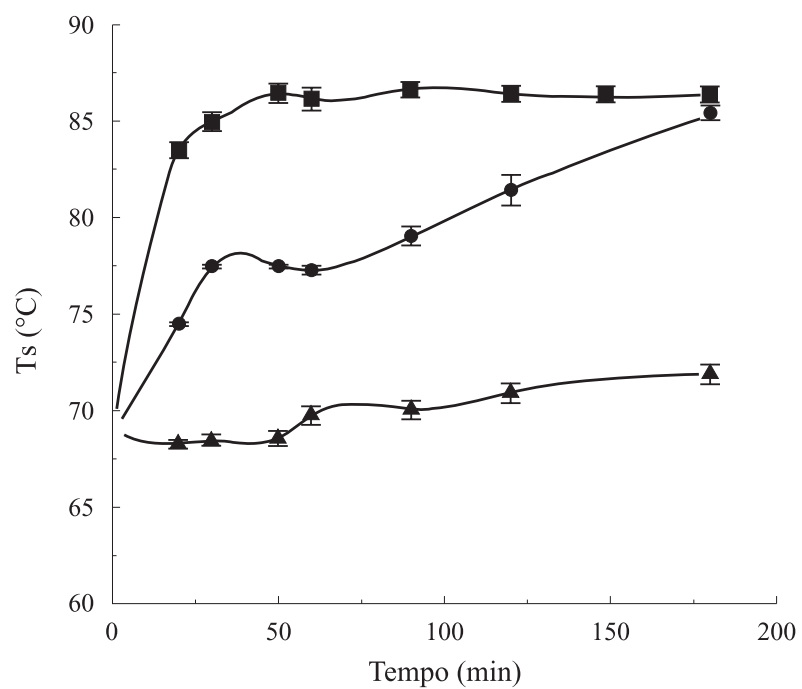

Figura 6. Comportamento de temperatura de encolhimento do pericárdio bovino pré-tratado com GA $0,001 \%$ por 3 h e reticulado seqüencialmente com glutaraldeído concentrações 0,005\% (- $\mathbf{\Delta}$-), $0,05 \%$ (-○-) e $0,5 \%$ (-口-). $\mathrm{O}$ ponto inicial das curvas corresponde ao do material após tratamento com GA $0,001 \%$. dos pontos de máximo e mínimo, principalmente quando se compara o perfil da curva após o tratamento com uma concentração de GA $0,5 \%$, concentração utilizada para a reticulação de GA no processo de confecção de válvulas cardíacas ${ }^{[15,21]}$.

Esta melhora do perfil da reatividade do PB com GA, em termos de estabilidade térmica foi confirmado pelo tratamento sequiencial do pericárdio bovino com concentrações de GA $0,001 \%$ e $0,005 \%$ por um período de $24 \mathrm{~h}$, seguido de tratamentos independentes com concentrações de GA $0,05 \%$ ou $0,5 \%$ (Figura 7). Nestes casos, os resultados mostraram um aumento rápido de Ts para valores superiores a $83,0^{\circ} \mathrm{C}$, aumento este que foi caracterizado por um perfil de evolução de estabilidade térmica também mais homogêneo, tanto em relação ao tratamento não seqüencial (Figura 3) quanto seqüencial realizados nas condições da Figura 6.

A verificação de que as curvas mais homogêneas em relação à estabilidade térmica são reflexos da melhoria da qualidade da reação, foi mostrada pela avaliação da estabilidade biológica do PB frente à colagenase e tripsina. Os resultados da Tabela 1 são aqueles para a hidrólise de PB tratado com colagenase com atividades de $36,2 \mathrm{U} / \mathrm{ml}$ e $72,5 \mathrm{U} / \mathrm{ml}$ para PB tratados com GA nas concentrações entre $0,001 \%$ a $0,5 \%$ (condições do experimento E) e inclui os valores de Ts das amostras de $\mathrm{PB}$, antes e após a reação de reticulação. Para a atividade de colagenase de 72,5 U/ml, a porcentagem de hidrólise para o PB nativo foi de $78,2 \%$ comparado com

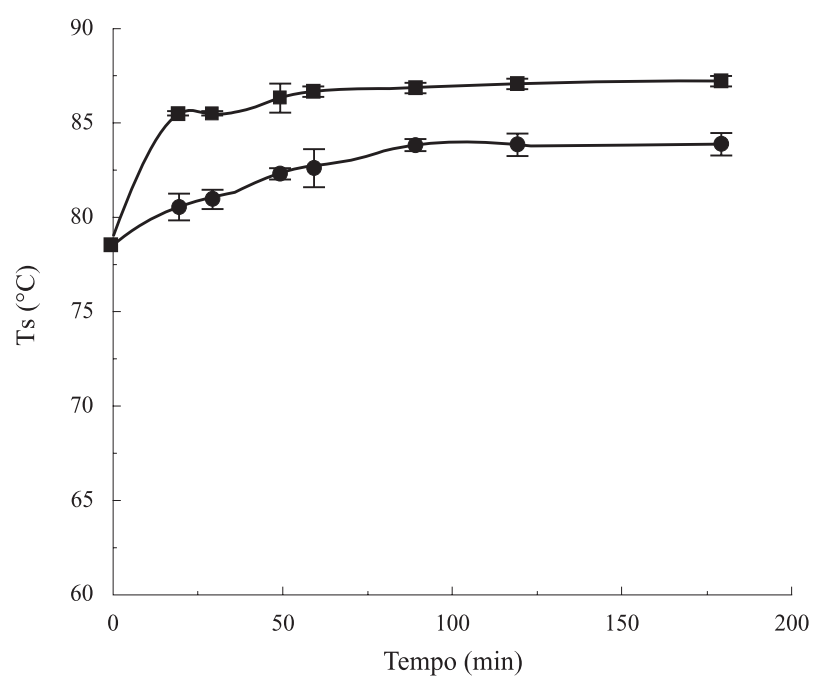

Figura 7. Comportamento de temperatura de encolhimento de pericárdio tratado seqüencialmente com GA $0,001 \%$ e $0,005 \%$ por tempos de $24 \mathrm{~h}$, e por $3 \mathrm{~h}$ nas concentrações de $0,05 \%$ (-) e e $0,5 \%$ (-口-). O ponto inicial das curvas corresponde ao material após o tratamento com GA 0,005\%. 
Tabela 1. Temperatura de encolhimento e percentual de hidrólise do pericárdio bovino tratado com colagenase em função da concentração de glutaraldeído (GA).

\begin{tabular}{cccc}
\hline & \multicolumn{2}{c}{ \% de Hidrólise ${ }^{(\mathrm{a})}$} & \\
\cline { 2 - 3 } Conc. de GA \% & \multicolumn{2}{c}{ Atividade Enzim. $(\mathbf{U} / \mathbf{m l})^{(\mathrm{b})}$} & Ts $\left({ }^{\circ} \mathbf{C}\right)$ \\
\hline 0 & 36,2 & 72,5 & \\
\hline 0,001 & 48,2 & 78,2 & 63 \\
0,005 & 23,0 & 33,5 & 68,9 \\
0,05 & 0,3 & 0,7 & 80,5 \\
0,5 & - & 0,5 & 80,5 \\
\hline
\end{tabular}

a) Determinada via Hydroxiprolina, após 72h de hidrólise.

b) Atividade calculada com base na preparação comercial $(435 \mathrm{U} / \mathrm{mg})$

$33,5 \%, 0,7 \%, 0,5 \%$ e $0,3 \%$ para os tratamentos com concentrações de GA a $0,001 \%, 0,005 \%, 0,05 \%$ e $0,5 \%$ respectivamente.

Embora os valores de Ts tenham sido na mesma ordem de grandeza, $63,1^{\circ} \mathrm{C}, 68,9^{\circ} \mathrm{C}, 80,5^{\circ} \mathrm{C}, 80,5^{\circ} \mathrm{C}$ e $86^{\circ} \mathrm{C}$, a atividade hidrolítica da colagenase só foi observada para PB tratados com uma concentração de GA de até $0,005 \%$. A partir desta concentração os resultados para a extensão da hidrólise foram todos próximos de $1 \%$, embora os valores de Ts variassem de 80 até $86,5^{\circ} \mathrm{C}$. Resultados similares foram observados quando os materiais foram tratados com uma atividade de enzima de 36,25 U/ml. Pelo fato de que nenhuma das duas concentrações de enzima utilizadas ter diferenciados materiais com estabilidade térmicas diferentes, principalmente para valores superiores a $80^{\circ} \mathrm{C}$, os resultados sugerem que Ts, um dos parâmetros empregado como um dos controles de qualidade de materiais destinados à confecção de válvulas, pode não ser adequado para este propósito.

A tripsina foi então empregada como método alternativo. Esta enzima atua somente sobre matrizes de colágeno desnaturado, com uma atividade específica para as ligações peptídicas adjacentes (na direção N-para o C-terminal) a resíduos de lisina, hidroxilisina e arginina (Figura 8). Entretanto, esta reação é inibida quando estes grupos estão bloqueados, o que ocorre com resíduos de lisina e hidroxilisina após a formação da base de Schiff (Figura 8). Assim sendo, a extensão da hidrólise por esta enzima é um indicador da participação destes dois aminoácidos na reação de reticulação.

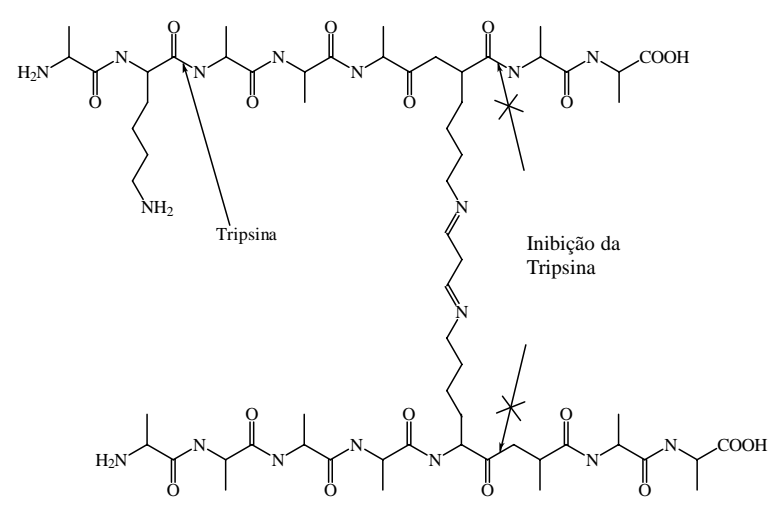

Figura 8. Diagrama esquemático da ação hidrolítica da tripsina sobre pericárdio bovino antes e após a reticulação com glutaraldeído.

Os resultados da hidrólise de PB reticulado com GA pela tripsina nas diferentes condições estudadas neste trabalho são mostrados na Figura 9. Enquanto que o material tratado seqüencialmente com concentrações de GA no intervalo entre 0,001 e $0,05 \%$ apresentou um grau de hidrólise de $2 \%$, o PB tratado com uma concentração pontual de $0,05 \%$ mostrou um grau de hidrólise cerca de $34 \%$, portanto uma estabilidade 17 vezes menor. Este aumento da estabilidade provavelmente não se deveu à maior concentração utilizada no método seqüencial, pois neste caso a concentração de GA total empregada foi de $0,056 \%$ (a soma das concentrações $0,001,0,005$ e $0,05 \%$ ) quando comparado com uma concentração de GA de $0,05 \%$ para o método não seqüencial.

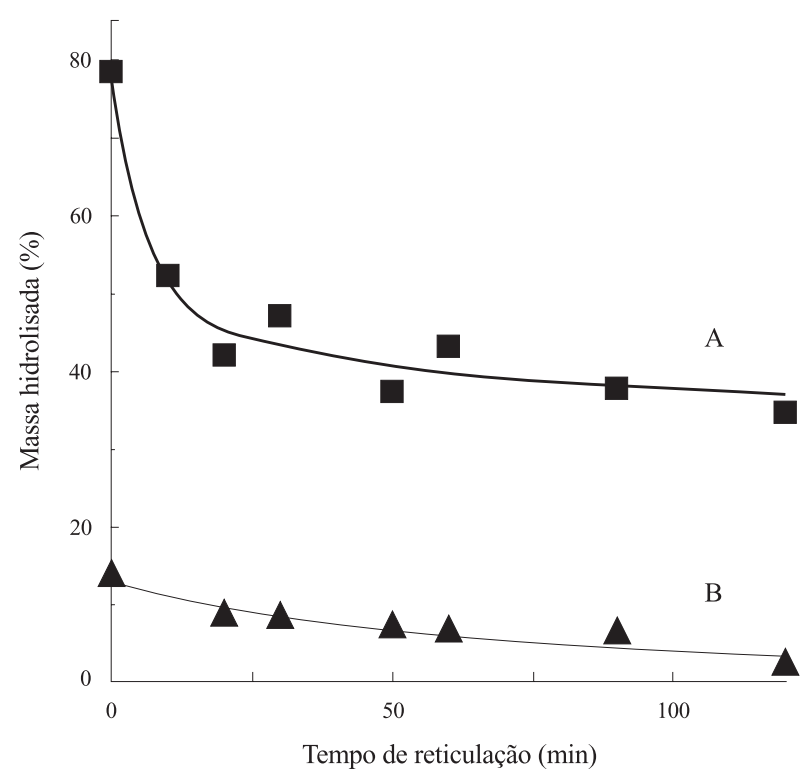

Figura 9. Hidrólise com tripsina do pericárdio bovino tratado com glutaraldeído: (a) $0,05 \%$; (b) $0,05 \%$, após tratamento seqüencial com GA a $0,001 \%$ e $0,005 \%$ por tempos de $24 \mathrm{~h}$. (o ponto zero desta curva corresponde ao material após o tratamento seqüencial). 
Tabela 2. Propriedades mecânicas para pericárdio bovino tratado seqüencialmente com concentrações de glutaraldeído no intervalo entre 0,001 e $0,05 \%$ em comparação com material tratado por método convencional.

\begin{tabular}{lcc}
\hline \multicolumn{1}{c}{ Parâmetros } & \multicolumn{2}{c}{ Tratamento } \\
\cline { 2 - 3 } & Convencional $^{\mathrm{b}}$ & Seqüencial \\
\hline Ts $\left({ }^{\circ} \mathrm{C}\right)$ & $83,0 \pm 1,2$ & $86,2 \pm 0,8$ \\
Espessura $(\mathrm{mm})$ & $0,25-0,30$ & 0,29 \\
Alongamento $(\%)$ & $>17$ & 60,5 \\
Índice de tenacidade & $>11,5$ & 34,3 \\
Resistência à traçao $\left(\mathrm{kgf} / \mathrm{mm}^{2}\right)$ & $>1,8$ & 1,1 \\
\hline
\end{tabular}

a) Ensaios realizados pela Braile Biomédica S.A.

b) Tratamento convencional com glutaraldeído utilizado na confecção de válvulas cardíacas ${ }^{[21]}$.

As propriedades mecânicas e elásticas do material tratado seqüencialmente em comparação com aquele tratado pelo método convencional são mostrados na Tabela 2. Observa-se que excetuando a resistência à tração, os outros parâmetros de desempenho mecânico encontram-se acima dos valores aceitáveis para PB utilizado na confecção de biopróteses $^{[21]}$. Entretanto, deve ser ressaltado que o material de comparação utilizados foi aquele pronto para a confecção da válvula, que passa por um tratamento convencional com GA com uma concentração cerca de 10 vezes maior $(0,5 \%)$ e por um período de 18 dias, com trocas constantes de solução durante este período ${ }^{[21]}$.

\section{Conclusões}

Os resultados acima sugerem que a reação de GA com tecidos naturais é heterogênea e está associada a dois fatores que são a complexidade do GA em solução e as variações estruturais da matriz colagênica à medida em que a reação de reticulação evolui. Variações na heterogeneidade da reação foram observadas também em função da concentração de GA. Os resultados de estabilidade enzimática frente à tripsina mostraram que o tratamento seqüencial é mais eficiente na promoção da reação de reticulação, produzindo em condições similares, materiais 17 vezes mais estáveis frente à hidrólise enzimática. Exceto pela resistência à tração, as propriedades mecânicas determinadas para materiais reticulados pelo método seqüencial foram superiores aos valores mínimos estabelecidos para PB utilizado na confecção de válvulas cardíacas. Apesar de não conclusivos, os ensaios com colagenase sugerem que o parâmetro temperatura de encolhimento (Ts) pode não ser adequado para controle de qualidade de materiais biológicos destinados à confecção de válvulas cardíacas. Os resultados mostram também que metodologia de reticulação seqüencial com GA, associada ao tratamento convencional, pode constituir-se em uma técnica de pré-tratamento promissora para a obtenção de materiais mais eficientes para confecção dispositivos cardiovasculares.

\section{Agradecimentos}

Os autores agradecem a Ezer Biazin e Glauco D. Broch pela assistência técnica. Ao PADCT/CNPq (contrato no 620001/96.8/FNM), FAPESP, Proc. no.1966/2455-8, e RHAE/CNPq (processo no 610.048/96-1) pelo apoio financeiro. Sônia D. Figueiró, da Universidade Federal do Ceará, foi bolsista CAPES-PICD, programa de mestrado junto à pós-graduação em Físico-química do IQSC-USP.

\section{Referências Bibliográficas}

1. Ionescu, M. I.; Ross, D. N. - Lancet, v.2, p.335-40 (1969).

2. Nimni, M. E. - J. Biomed. Mater. Res., v..35, p.53137 (1997).

3. Lee, Y. S. - J. Electron Microsc., v.43, p.131-40 (1994).

4 Bernacca, G. M.; Fisher, A.C.; Wilkinson, R.; Mackay, T.G.; Wheatley, D.J. - J. Biomed. Mater. Res., v.26, p.959-66 (1992).

5. Schoen, F. J.; LEVY, R. J. - Eur. J. Cardiothorac. Surg., v.6, S91-3 (1992).

6. Schoen, F.J., Tsao, J.W., Levy, R.J. - Am. J. Pathol. v.23,123-45 (1995).

7. Sung, H. W.; Tu, R.; Shen, S. H.; Witzel, T. H.; Lin, D.; Hata, C.; Kingsbury, C. J.; Noishiki, Y.; Tomizawa, Y.; Quijano, R. C. - Asaio J., v.40, p.192-8 (1994).

8. Petite, H.; Frei, V.; Huc, A.; Herbage, D. - J. Biom. Mat. Res., v.28, p.159-65 (1994).

9. Naimark, W. A.; Pereira, C. A.; Tsang, K.; Lee, J. M. - J. Mat. Sci.: Mat. in Med., v.6, p.235-41 (1995). 
10. Lee, J. M.; Edwards, H. H. L.; Pereira, C. A.; Samii, S. I. - J. Mat. Sci.: Mat. in Med., v.7, p.531-41 (1996).

11. Gott, J. P.; Giratdot, M. N.; Girardot, J. M.; Hall, J. D.; Whitlark, J.D.; Horsley, W. S.; Dorsey, L. M.; Levy, R. J.; Shoen, F. J.; Guyton, R. A. Ann. Thorac. Sug., v. 64, p. 50-58 (1997).

12. Song, T.; Vesely, L.; Boughner, D. - Biomaterials, v.22, p.89-98 (1990).

13. Ruidgrok, J. M.; Wijn, J. R.; Boon, M. E. - J. Mat. Sci. Mat. Med., v.5, p.80-7 (1994).

14. Goissis, G.; Yoshioka, S. A.; Braile, D. M.; Ramirez, W. D. - Artif. Organs, In press

15. Chapman, J. A.; Tzaphlidou, M.; Meek, K. M. Electron. Microsc. Ver., v.3, p.143-82 (1990).

16. Liao, K. X.; Frater, R. W.; Stevenson, R.; Smith, W.; Nikolic, S. D., Macaluso, F.; Yellin, E. L. Asaio Trans., v.37, M349-M351 (1991).

17. Woodroof, A. - J. Bioeng., v.2, p.1-19 (1978).

18. Cheung, D. T.; Nimni, M. E. - Connect. Tissue Res., v.13, p.109-15 (1985).

19. Cheung, D. T.; Natashia, P.; Ko, E. C.; Nimni, M. E. - Connect Tis. Res., v.10, p.201-16 (1982).

20. Rao, K. P.; Joseph, T. - "Graft copolymers and their biomedical applications", In: Nimni, ME,
Ed. Collagen (Vol. III. Biotechnology), Boca Raton, FL: CRC Press Inc., p. 63-86 (1988)

21. Braile, D. M. - "Prótese valvular de pericárdio bovino: desenvolvimento e aplicação clínica na posição mitral", Tese de Doutorado, Escola Paulista de Medicina, São Paulo (1990)

22. Simmons, D. M.; Kearney, K. - Biotechnol. Appl. Biochem., v.17, p.23-9 (1993).

23. Grabenwoger, M.; Grimm, M.; Eybl, E.; Leukauf, C.; Muller, M. M.; Plenck Jr., H.; Bock, P. - J. Biomed. Mater. Res., v.26, p.1231-40 (1992).

24. Stegemann, H. H. ; Stalder, K. - Clin. Chim. Acta, v.18, p.267-272 (1967).

25. Bruening, G. - Biochemical Experiments., John Wyley, Cap. 1, p. 1-11, New York (1970).

26.Kopp, K.; Bonnet, M.; Renou, J. P. - Matrix, v 9, p.443-51 (1989).

27. Van Noort, J.; Yates, S. P.; Martin,T. R. P.; Barker, A. T.; Black, M. M. - Biomaterials, v.3, p. 21-6 (1982)

28. Liao, K.; Frater, R. W.; Lapietra, A.; Ciuffo, G.; Ilardi, C. F.; Seifter, E. - Ann. Thorac. Surg., v.60, S343-S347 (1995).

Recebido: 19/08/97 Aprovado: 24/03/98 